\title{
Sternoclavicular Joint Distances and Degenerative Changes in Computed Tomography
}

\author{
Vefa Çakmak' (D), Mert Özen² (D) \\ 'Department of Radiology, Pamukkale University Faculty of Medicine, Denizli, Turkey \\ ${ }^{2}$ Department of Emergency Medicine, Pamukkale University Faculty of Medicine, Denizli, Turkey \\ ORCID iDs of the authors: V.ç. 0000-000I-7002-5594; M.Ö. 0000-000I-6653-3756.
}

Cite this article as: Çakmak V, Özen M. Sternoclavicular Joint Distances and Degenerative Changes in Computed Tomography. Cyprus J Med Sci 2021; 6(2): I36-140.

\section{BACKGROUND/AIMS}

To evaluate sternoclavicular joint distances and age-related degenerative changes in thorax computed tomography images.

\section{MATERIAL and METHODS}

Computed tomography (CT) was performed between April 2019 and October 2019 at the Department of Radiology of State Hospital for chest pain and lung parenchymal nodule follow-up. One hundred and forty-six sternoclavicular joints of 73 patients (38 males, 35 females, median 58 years, mean $57.23 \pm 13.25$ years) aged 35-78 years were evaluated retrospectively. Measurements were taken for bilateral sternoclavicular joint from anterior, mid, and posterior in the axial plain and superior, mid, and inferior sections in the coronal plan. Measurements of sternoclavicular joint were compared according to gender for right and left sides. The presence of osteophyte, sclerosis, subchondral cyst, and gas showing degeneration in the sternoclavicular joint were compared according to age and sex.

\section{RESULTS}

Sternoclavicular joint distances measured in axial and coronal planes were found to be significantly lower in female gender group than in men. In the study group, there was statistically significant difference between the measurements made from the coronal plan superior section $(P=.016)$. There was no statistically significant difference between mid and inferior measurements in coronal plane and anterior, mid and posterior measurements in axial plane. Sternal osteophytes were significantly more common in females than males $(P=$ .021). Osteophyte located in the clavicle was significantly more frequent with increasing age.

\section{CONCLUSION}

There is no significant asymmetry in the sternoclavicular joint in asymptomatic individuals, and an increase in degenerative markers of the sternoclavicular joint is observed with the progression of age.

Keywords: Computed tomography, sternoclavicular joint, osteophyte

\section{INTRODUCTION}

Sternoclavicular joint is a diarthrodial joint located between the upper extremity and axial skeleton. Joint cartilage is composed of fibrous and hyaline cartilage intertwined with each other. Anterior and posterior sternoclavicular ligaments, costoclavicular ligament, and interclavicular ligament form the ligamentous part of the sternoclavicular joint., ${ }^{1,2}$ Apart from traumatic conditions, rheumatological disorders, infectious disorders, calcium pyrophosphate deposit disease, and degenerative disorders also affect the joint. 3,4 Osteoarthritis does not cause joint instability. 2,5 Computed tomography (CT) is widely used for traumatic and rheumatological conditions affecting the joint. ${ }^{6-8}$ A number of studies have investigated the normal anatomic properties and variations of the sternoclavicular joint., 90 This study aimed to assess degenerative changes affecting the sternoclavicular joint spaces and the joint itself in computed tomographic imaging.

\section{MATERIAL and METHODS Study Group}

This study was approved by the regional Ethics Committee of Pamukkale University Faculty of Medicine (no: 60116787020/I8I0). Sternoclavicular joint properties were retrospectively analyzed on CT images of I38 patients undergoing CT 


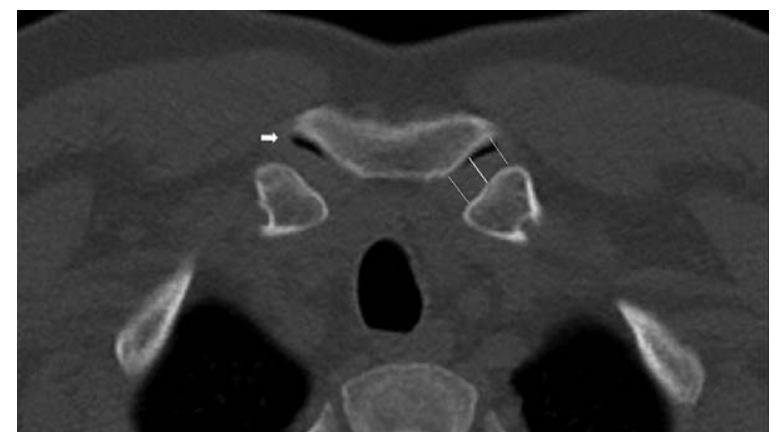

Figure I. CT image of the sternoclavicular joint in the axial plane. Gas is present in both SCJ joints (white arrow indicates gas in right SCJ). The left SCJ measurements were made from the anterior, mid, and posterior section(lines)

for various reasons such as chest pain or parenchymal lung nodule monitoring at State Hospital, Department of Radiology between April 2019 and October 2019. Among those patients, the CT images of those with thoracic roto-scoliosis, history of bypass surgery or severe chest trauma, sternoclavicular anomaly, oncological disorders, or metabolic bone disease were excluded from the analysis. One hundred and forty-six sternoclavicular joints of 73 patients aged $35-78$ years (38 males, 35 females, median age 58 years, mean age $57.23 \pm 13.25$ years) were retrospectively analyzed.

\section{Computerized Tomography}

CT was performed with a l6-detector row multi-slice helical CT device (I6 MDCT CT scanner, Toshiba Alexion, Japan). All thoracic $C T$ examinations were performed over ascenogram image, by scanning the region between the neck and upper pole of kidney, with the patients lying in supine position and their hands resting on both sides of the head. The imaging parameters were as follows: tube voltage $120 \mathrm{kV}$, tube current $70 \mathrm{mAs}$, slice thickness $2.0 \mathrm{~mm}$, rotation time $750 \mathrm{~ms}$, and "pitch" 0.938. All CT images were analyzed at mediastinal (WW:350, WL:50), parenchymal (WW:-600, WL:1600), and bone (WW:2500, WL:480) windows at the workstation.

\section{Image Analysis}

Bilateral sternoclavicular joint distances of patients enrolled between April 2019 and October 2019 were measured on axial

\section{Main Points}

- The sternoclavicular joint is a diarthrodial type joint composed of cartilage and ligament complex between the upper extremity and the axial skeleton.

- Sternoclavicular joint instability is often caused by rheumatological diseases and trauma.

- Sternoclavicular joint distances are observed as lower in women compared to men.

- The frequency of osteophytes in the clavicle increases with aging.

- Gas in the sternoclavicular joint is more common in 35-49 years and men.

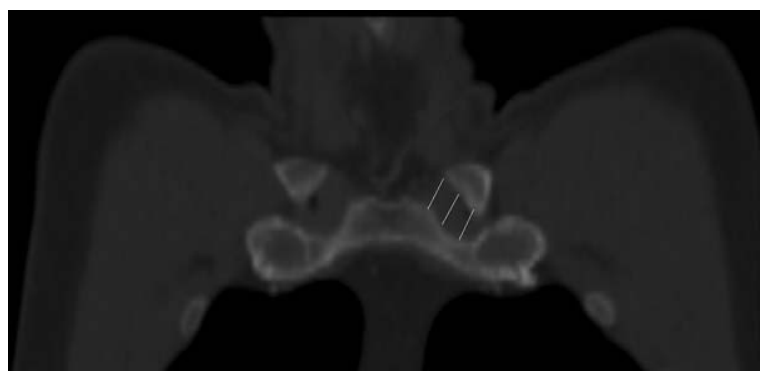

Figure 2. CT image of the sternoclavicular joint in the coronal plane. The left SCJ measurements were made from the superior, mid, and inferior section(lines)

and coronal planes on CT images. Measurements were made from the superior, mid, and inferior aspects on axial plane and from the anterior, mid, and posterior aspects on coronal plane for both joints (Figures I and 2). Measurements of the sternoclavicular joint were compared between the right and left sides by gender. The study population was grouped by age range. Osteophytes, sclerosis, subchondral cyst, and gas, all of which indicate sternoclavicular joint degeneration, were compared by age range and sex

\section{Statistical Analysis}

Data analysis was performed using a Statistical Package for the Social Sciences (SPSS) version 2I (IBM SPSS Corp.; Armonk, NY, USA). Descriptive statistics included mean \pm standard deviation for continuous variables and \% for categoric variables. Chi-square test was used to compare categoric variables. Comparison of two sternoclavicular joint spaces on axial and coronal planes was performed with t test. $P<.05$ was considered statistically significant.

\section{RESULTS}

Sternoclavicular joint space distances measured on coronal and axial planes from CT images are presented in Table I. Sternoclavicular joint distances were significantly lower in women compared to men. In measurements made from the superior aspect on coronal plane, mean sternoclavicular joint distance was $11.37 \pm 2.97 \mathrm{~mm}$ on the right side and $10.87 \pm 2.89 \mathrm{~mm}$ on the left side. There was a statistically significant difference between measurements made from the superior aspect on coronal plane $(P=.016)$. The measurements made from the mid and inferior aspects on coronal plane and the measurements made from the anterior, mid, and posterior aspects on axial plane showed no significant difference between both sides. Seventy-three patients whose CT images were analyzed were grouped by age. Accordingly, there were 24 patients aged $35-$ 49 years, 15 patients aged 50-59 years, 19 patients aged 60-69 years, and 15 patients aged 70 years or older. Degenerative signs in the sternoclavicular joint were compared by age range and sex (Table 2) (Figure 3). Women had a significantly higher rate of osteophytes located in the sternum ( $P=.021$ ) (Figure 4). The rate of clavicular osteophytes significantly showed a significant increase with advancing age. Presence of gas was significantly more common in patients aged 35-49 years and men compared to women (Table 3). Five patients were found to have sternal ossicle, and seven patients had clavicular notch (Figure 5). 


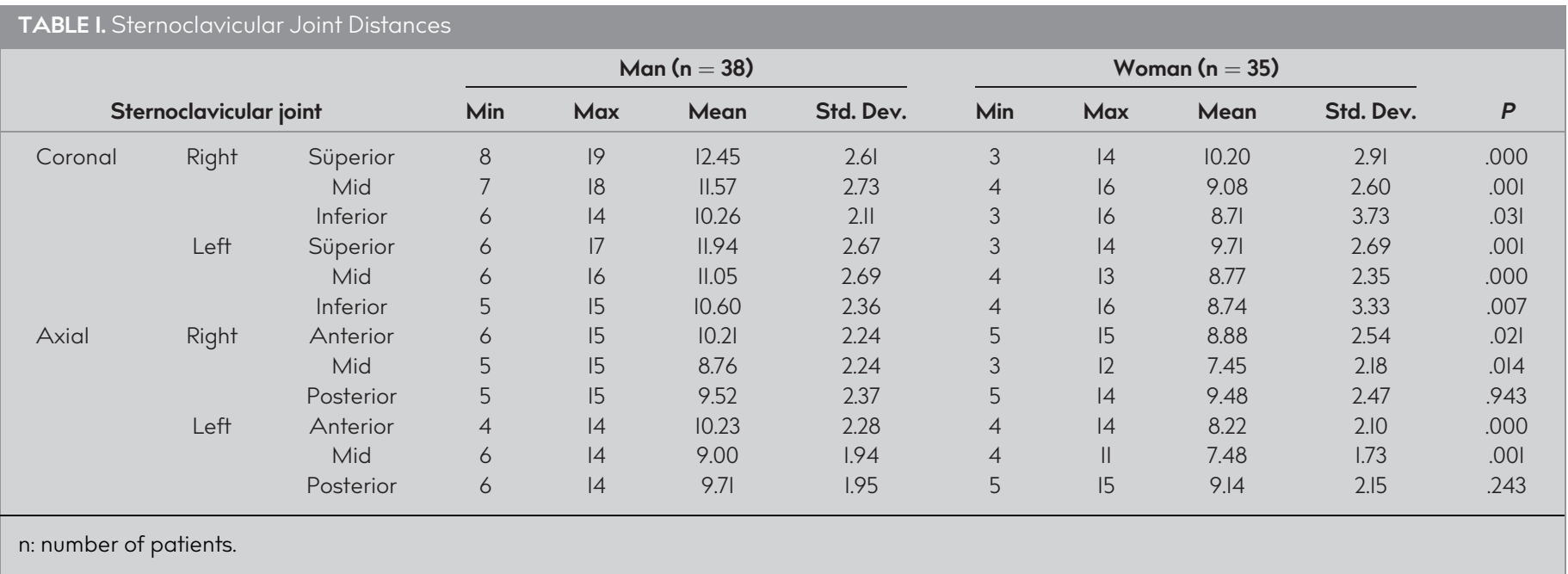

TABLE 2. Markers of Degeneration in the Sternoclavicular Joint

\begin{tabular}{|c|c|c|c|c|c|c|}
\hline & \multicolumn{2}{|c|}{ Osteophytes } & \multicolumn{2}{|c|}{ Sclerosis } & \multicolumn{2}{|c|}{ Subchondral cyst } \\
\hline & Clavicle & Sternum & Clavicle & Sternum & Clavicle & Sternum \\
\hline Man & 10 & 2 & 3 & 2 & 4 & 2 \\
\hline$P$ & .214 & .021 & .104 & .418 & .334 & .294 \\
\hline $35-49$ years & 0 & 2 & । & I & I & I \\
\hline 50-59 years & 6 & 2 & 3 & 2 & 2 & 0 \\
\hline$P$ & .000 & .164 & .219 & .280 & .224 & .289 \\
\hline
\end{tabular}

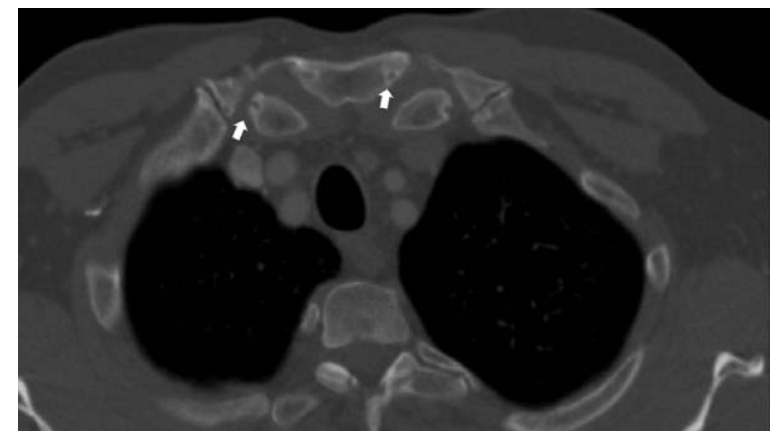

Figure 3. Sternal and clavicular subchondral cysts (white arrows)

\section{DISCUSSION}

Standard radiography cannot provide adequate detail for the assessment of sternoclavicular joint. Brossmann et al." provided anatomic details of joint cartilage of the sternoclavicular joint using magnetic resonance imaging (MRI). MRI has a limited use for the evaluation of the sternoclavicular joint due to respiratory and pulse artifacts. Several studies have used ultrasonography to demonstrate joint effusion and infectious conditions involving the sternoclavicular joint. ${ }^{4} \mathrm{CT}$ is a widely used imaging modality in routine practice to detect abnormalities of the sternoclavicular joint, which do not appear in plain radiograms.

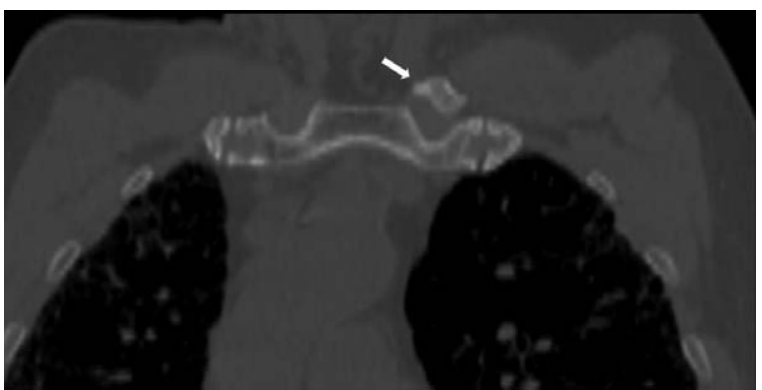

Figure 4. Left clavicular osteophyte (white arrow)

A study that examined the high-resolution CT properties of the sternoclavicular joint showed that all individuals older than 50 years had degenerative changes affecting the joint. ${ }^{12}$ It was reported that people of advanced age had a greater rate of osteophytes, subchondral cysts, and sclerosis. ${ }^{8}$ Our study also demonstrated an aging-related increase in the prevalence of signs of degeneration, such as osteophytes, subchondral cysts, and sclerosis.

We detected a smaller sternoclavicular joint distance on both sides in women compared to men. When the whole study group was concerned, we detected a $\sim 0.5 \mathrm{~mm}$ difference 
TABLE 3. Gas in the Sternoclavicular Joint

\begin{tabular}{|ccccccc|} 
& & & \multicolumn{3}{c}{ Age range } \\
\cline { 5 - 7 } Gas in the sternoclavicular joint & Man & Woman & $\mathbf{3 5 - 4 9}$ years & $\mathbf{5 0 - 5 9}$ years & $\mathbf{6 0 - 6 9}$ years & $\mathbf{7 0}$ years and older \\
\hline Positive & 21 & 10 & 18 & 5 & 6 & 2 \\
Negative & 17 & 25 & 6 & 10 & .001 & 13 \\
$P$ & & .033 & & & 13 \\
\hline
\end{tabular}

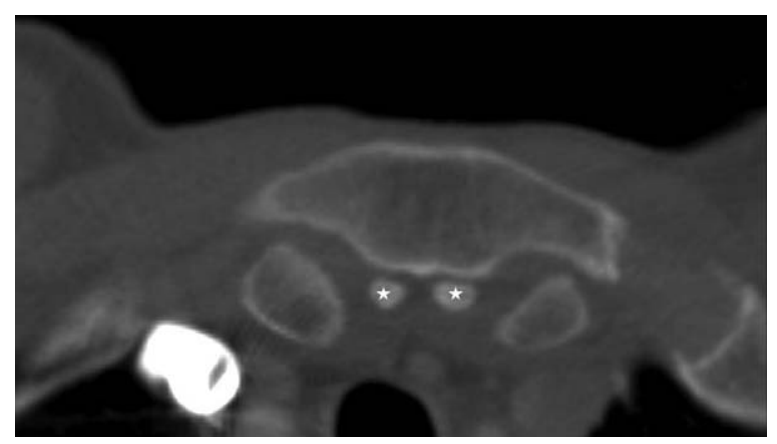

Figure 5. CT image of the sternal ossicle in the axial plane (stars)

between sternoclavicular joint distances in measurements made from the superior aspect on coronal plane. We did not detect any asymmetry between both sides in measurements made from the mid and inferior aspects on coronal plane and in measurements made from the anterior, mid, and posterior aspects on axial plane. We believe that this was caused by the patients' arms being positioned on both sides of the head. Tuscano et al., ${ }^{9}$ in a study examining the sternoclavicular joint in axial thoracic CT images of 104 patients, reported that there may be asymmetry of up to $5.7 \mathrm{~mm}$ between the sternoclavicular joints on both sides. The present study also used thoracic CT images, and we believe that asymmetry resulted from patient position. Another study retrospectively examined the sternoclavicular joint in carotid CT angiography images, and it showed symmetry in both joints. ${ }^{10}$ We believe that it stemmed from neutral arm position. Celikyay et al. ${ }^{8}$ reported a lesser amount of reduction in joint distance in the control group than the patient group with rheumatoid arthritis. De Maeseneer et al. ${ }^{10}$ reported that joint distance tended to decrease with advancing age and joint distance may be minimally reduced with aging. Sternoclavicular joint asymmetry was reported to cause a tendency for osteoarthritis and joint instability. ${ }^{2}$

We detected osteophytes, subchondral cysts, and sclerosis on clavicular and sternal aspects of the sternoclavicular joint. The prevalence of these degenerative signs increased with advancing age. We found a significantly higher prevalence of clavicular osteophytes among patients aged 70 years or older. We identified degenerative signs more commonly on the sternal aspect than the clavicular aspect. We believe that this finding is attributable to motion of the clavicular head with arm movements. Baker et al. ${ }^{12}$ detected sclerosis and subchondral cysts on the outer clavicular surface more commonly than the joint surface in almost $50 \%$ of the study population. However, another study reported that osteophytes and subchondral cysts were located at equal rates on clavicular and sternal tips. $^{10}$
Baker et al. ${ }^{12}$ reported that there was diffuse intraarticular gas content within the sternoclavicular joint. Tuscano et al. ${ }^{9}$ detected intraarticular gas in $32 \%$ of patients, with $8 \%$ having bilateral gas content. We found that women had a greater rate of intraarticular gas content than men. On the other hand, we revealed that gas content was reduced by advancing age. We also showed that while there occurred a reduction in gas content, degenerative signs increased.

Shirazian et al. $^{3}$ reported a rate of $17 \%$ for chondrocalcinosis within the sternoclavicular joint. Another study reported a rate of $3 \%$ for the same condition. ${ }^{10}$ We did not examine the presence of calcification within the sternoclavicular joint. We detected sternal ossicles in five patients and clavicular notch in seven patients.

Sternoclavicular joint subluxations are known to be rare and have difficulties in treatment. In patients with subluxation, we think the joint distances in this study will help with diagnosis. However, sternoclavicular joint distance and asymmetry of the joint show importance in arthroscopy in insertion surgical approach. $^{13}$

Our study had some limitations. The major one is area small sample volume and a single-center design. Another limitation was its retrospective nature. We did not assess the soft tissue component of the sternoclavicular joint when we examined sternoclavicular joint distance and degenerative changes affecting the joint. As patients' hands were technically positioned at the level of head during the acquisition of CT images, we found a significant difference in the measurements made from the superior aspect on coronal plane. Therefore, we believe that using CT images acquired when both arms on both sides would be more useful for the evaluation of the sternoclavicular joint.

In conclusion, women have a narrower sternoclavicular joint distance than men, and sternoclavicular joint of healthy people does not show marked asymmetry. The study prevalence of degenerative signs of the sternoclavicular joint increased with advancing age. Gas content of the sternoclavicular joint is reduced with aging.

Ethics Committee Approval: Ethical committee approval was received from the Pamukkale University Faculty of Medicine Ethics Committee (PAÜ 60116787-020/18110).

\section{Informed Consent: N/A}

Peer-review: Externally peer-reviewed.

Author Contributions: Concept - V.Ç., M.Ö.; Design - V.Ç.; Supervision V.Ç.; Resource - V.Ç., M.Ö.; Materials - V.Ç., M.Ö.; Data Collection and/ or Processing - V.Ç., M.Ö.; Analysis and/or Interpretation - V.Ç., M.Ö.; Literature Search - V.Ç.; Writing - V.Ç.; Critical Reviews - V.Ç., M.Ö. 
Conflict of Interest: The authors have no conflicts of interest to declare.

Financial Disclosure: The author declared that this study has received no financial support.

\section{REFERENCES}

I. Frosi G, Sulli A, Testa M, Cutolo M. The sternoclavicular joint: Anatomy, biomechanic, clinical features and aspects of manual therapy. Reumatismo. 2004;56:82-88.

2. Sewell MD, Al-Hadithy N, Le Leu A, Lambert SM. Instability of the sternoclavicular joint: Current concepts in classification, treatment and outcomes. Bone Joint J. 2013;95(6):721-731. [CrossRef]

3. Shirazian H, Chang EY, Wolfson T, Gamst AC, Chung CB, Resnick DL. Prevalence of sternoclavicular joint calcium pyrophosphate dihydrate crystal deposition on computed tomography. Clin Imaging. 2014;38(4):380-383. [CrossRef]

4. Johnson MC, Jacobson JA, Fessell DP, Kim SM, Brandon C, Caoli E. The sternoclavicular joint: Can imaging differentiate infection from degenerative change? Skeletal Radiol. 2010;39:551-558. [CrossRef]

5. Lawrence CR, East B, Rashid A, Tytherleigh-Strong GM. The prevalence of osteoarthritis of the sternoclavicular joint on computed tomography. J Shoulder Elbow Surg. 2017;26:18-22.

6. Ernberg L, Potter H. Radiographic evaluation of the acromioclavicular and sternoclavicular joints. Clin Sports Med. 2003;22(2):255275. [CrossRef]
7. Lucet L, Le Loët X, Ménard JF, et al. Computed tomography of the normal sternoclavicular joint. Skelet Radiol. 1996;25:237-24l. [CrossRef]

8. Celikyay F, Yuksekkaya R, Inanir A, Deniz C. Multidetector computed tomography findings of the sternoclavicular joint in patients with rheumatoid arthritis. Clin Imaging. 2013;37(6):II04-II08. [CrossRef]

9. Tuscano D, Banerjee S, Terk MR. Variations in normal sternoclavicular joints: A retrospective study to quantify SCJ asymmetry. Skeletal Radiol. 2009;38:997-I00I. [CrossRef]

10. De Maeseneer M, Lenchik L, Buls N, et al. High-resolution CT of the sternoclavicular joint and first costochondral synchondrosis in asymptomatic individuals. Skeletal Radiol. 2016;45:1257-I262. [CrossRef]

II. Brossmann J, Stäbler A, Preidler KW, Trudell D, Resnick D. Sternoclavicular joint: MR imaging: Anatomic correlation. Radiology. 1996;198(I):193-198. [CrossRef]

12. Baker ME, Martinez S, Kier R, Wain S. High resolution computed tomography of the cadaveric sternoclavicular joint: Findings in degenerative disease. J Comp Tom. 1988;I2(I):I3-18. [CrossRef]

13. Van Tongel A, Van Hoof T, Pouliart N, Debeer P, D'Herde K, De Wilde L. Arthroscopy of the sternoclavicular joint: An anatomic evaluation of structures at risk. Surg Radiol Anat. 2014;36:375-38I. 